\title{
ANÁLISE DE PRÁTICAS HIBRIDAS DE GERENCIAMENTO DE PROJETOS EM UMA EMPRESA DE SOFTWARE
}

Michael Jordan Bianchi (michael_bianchi@usp.br) - Escola de Engenharia de São Carlos, Universidade de São Paulo.

Daniel Capaldo Amaral (amaral@usp.br) - Escola de Engenharia de São Carlos, Universidade de São Paulo.

\section{RESUMO}

As organizações estão se esforçando para desenvolver produtos de forma mais rápida, por meio de processos mais flexíveis. Um dos desafios atuais é a necessidade de ajustar as práticas de gerenciamento para diferentes tipos de projeto e segmentos de mercado. Uma das soluções tem sido a combinação de práticas de gestão de projetos provenientes da teoria tradicional e ágil, e esse fenômeno vem sendo denominado de modelos híbridos de gerenciamento. Este trabalho analisa as práticas de gestão de projetos em uso em uma empresa de softwares que desenvolve projetos utilizando ambas as abordagens (tradicional e ágil) e compara-as com os modelos híbridos presentes na literatura. O método escolhido foi o estudo de caso, onde foram entrevistadas 4 equipes de desenvolvimento responsáveis por diferentes tipos de projetos dentro da organização. A pesquisa identificou similaridades e diferenças em relação aos modelos da literatura e aponta um potencial problema em relação aos modelos observados. É indicado que neste tipo de empresa a adequação das práticas deva ser realizada para cada projeto, de maneira específica, ao invés da existência de um modelo de gestão geral para utilizar em quaisquer projetos da empresa. Este resultado demonstra que a estratégia de se criar um modelo híbrido único e geral pode não ser a melhor alternativa neste tipo de organização.

Palavras chave: Gerenciamento de projetos; Gerenciamento ágil de projetos; Gestão híbrida de projetos; Modelos híbridos de gestão.

Área: Gestão do Processo de Desenvolvimento de Produtos 


\section{INTRODUÇÃO}

Um dos principais desafios em gerenciamento de projetos é a necessidade de ajustar os modelos de gestão, por meio da adequação das práticas de gerenciamento para diferentes tipos de projeto e segmentos de mercado, devido às especificidades de cada caso, como evidenciado em de Ktata e Lévesque (2009), Conforto et al., (2014) e Conforto, Rebentisch e Amaral (2014).

Há na teoria de projetos práticas ditas ágeis e outras orientadas ao plano, conhecidas como tradicionais. Empresas de diferentes setores da economia, porém, demonstram dificuldades na aplicação dessas práticas de forma pura. Nos últimos anos vem crescendo a ideia de combinação entre práticas de diferentes abordagens, gerando os chamados modelos híbridos de gestão (SILVA; AMARAL, 2014).

Esses modelos seriam provenientes da necessidade de inovar no gerenciamento dos projetos para solucionar os novos desafios impostos pelo mercado, com o objetivo de garantir o sucesso das organizações, conforme pode ser evidenciado em trabalhos recentes como Sommer et al. (2015), Silva (2015), Conforto e Amaral (2016) e Cooper (2016).

Apesar de todo interesse em volta desses modelos, um dos principais desafios é entender como utilizá-los dentro da realidade de uma empresa. Diversos autores propõem diferentes modelos híbridos de gestão de projetos (NAWROCKI et al., 2006; SEYAM; GALAL-EDEEN, 2011; AMBLER, 2013; COOPER, 2014; CONFORTO; AMARAL, 2016; SOMMER et al., 2015). A questão entretanto é analisar e entender se esses modelos híbridos refletem a realidade enfrentada pelas empresas que estão adotando tais práticas.

Esta pesquisa investigou o assunto por meio de um levantamento de práticas relacionadas ao planejamento e controle de escopo e tempo de projetos em uma empresa de serviços de tecnologia da informação, a qual trabalha com desenvolvimento de softwares personalizados, além de serviços gerenciados e projetos de TI. A empresa possui experiência em práticas ágeis e realiza projetos envolvendo ambas as abordagens, com escopo fechado e ágil.

Pelo fato de conviver com projetos de diferentes características, a empresa utiliza modelos de gerenciamento personalizados, realizando a combinação de práticas de acordo com o tipo de projeto, a fim de satisfazer suas especificidades, bem como as expectativas de seus clientes. $\mathrm{O}$ objetivo foi comparar as práticas em uso na organização com os modelos híbridos encontrados na literatura, verificando similaridades e diferenças, a fim de discutir a utilidade desses modelos e identificar questões de pesquisa futura na área.

\section{REVISÃO DA LITERATURA}

\subsection{Gerenciamento Híbrido de Projetos}

As abordagens ágil e tradicional de gerenciamento de projetos são comumente interpretadas como alternativas opostas e apropriadas para tipos diferentes de projetos. Segundo a teoria, a abordagem ágil seria aplicável apenas em casos que envolvam projetos inovadores, equipes pequenas e co-localizadas, enquanto a abordagem tradicional seria aplicável em projetos complexos, com produtos e condições de maior previsibilidade (BOEHM, 2002; BATRA et al., 2010).

Estudos recentes demonstraram que diferentes organizações de vários setores da economia utilizam elementos de ambas as abordagens, misturando práticas, apesar de declararem a opção por uma abordagem em específico (Conforto et al., 2014). Além disso, dados demonstram que quando em ambientes grandes e complexos a inovação é envolvida, há dificuldade em se utilizar práticas ágeis puras, devido ao tamanho da equipe de projeto, muitas vezes distribuídas 
geograficamente, tendo a necessidade de se documentar o projeto para não comprometer o mesmo (CONFORTO et al., 2015).

Segundo Batra et al., (2010) agilidade sem um processo estruturado pode levar ao chamado "caos", especialmente em ambientes grandes e complexos, onde o planejamento e controle são fatores críticos para o sucesso do projeto, enquanto que a estrutura sem agilidade pode levar à rigidez, afetando de forma negativa o projeto. Nesse cenário, a habilidade de criar modelos híbridos e adaptá-los se faz necessário para as empresas e profissionais da área de gerenciamento de projetos (CONFORTO et al., 2015).

O uso de modelos híbridos tem sido considerado na literatura como a principal opção para este problema, como comprovado em diversos trabalhos na área (BOEHM; TURNER, 2003; BATRA et al., 2010; BARLOW et al., 2011; CONFORTO; AMARAL, 2016).

Segundo Galal-edeen, Riad e Seyam (2007) modelos híbridos podem ser definidos como a combinação das abordagens para encontrar um meio termo que combine as vantagens e corrija as deficiências de ambas (tradicional e ágil). Tais modelos portanto, mesclam as práticas das abordagens de gestão de projetos, a fim de equilibrar a flexibilidade e produtividade com a previsibilidade e procedimentos da organização, visando a entrega de melhores resultados para o cliente.

Trabalhos anteriores identificaram que uma das condições críticas para o uso de modelos híbridos está no processo de adaptação das práticas para diferentes tipos de projetos (CONFORTO; AMARAL, 2016, p.12). Essa adaptação depende das características do projeto e do ambiente em que está inserido, devendo estar alinhada com as necessidades do projeto de seus clientes.

Apesar das vantagens identificadas, grande parte dos estudos voltados para esse tema foca no desenvolvimento e/ou aplicação de modelos híbridos predefinidos em empresas específicas, não havendo discussão sobre a utilidade/usabilidade desses modelos em diferentes realidades e setores da economia (COBB, 2012; SILVA, 2015; SOMMER et al., 2015; CONFORTO; AMARAL, 2016). Um problema adicional é o fato de existirem organizações que convivem com projetos de diferentes características e que muitas vezes precisam ser gerenciados por meio de abordagens distintas por exigência de seus clientes. Como fica a adoção de modelos híbridos nestas organizações, dado que uma solução específica pode não valer para todas as condições?

\section{MÉTODO}

O método escolhido foi o estudo de caso (MIGUEL, 2007; YIN, 2001). Segundo Yin (2001), o estudo de caso é indicado para estudos que pretendem compreender como e por que certas decisões são tomadas. Dentre os benefícios de um estudo de caso está a possibilidade do desenvolvimento de novas teorias, aumento do entendimento sobre eventos reais e contemporâneos (MIGUEL et al., 2011).

A realização do estudo de caso ocorreu de acordo com as atividades ilustradas na Figura 1 e descritas a seguir. 
Figura 1. Atividades do estudo de caso. Fonte: Autoria própria.

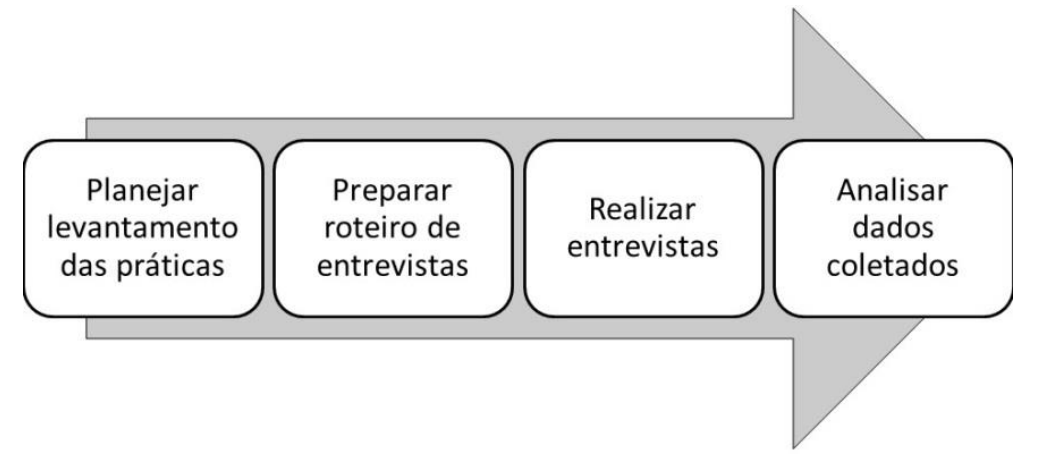

Planejar levantamento das práticas de gerenciamento de projetos em uso na empresa. Nessa atividade foi definido que o levantamento das práticas da organização seria realizado por meio da técnica de entrevistas (MARCONI; LAKATOS, 2003) com os gerentes e líderes dos projetos selecionados, utilizando um roteiro de entrevistas semiestruturado como guia. Foram entrevistadas 4 equipes de desenvolvimento responsáveis por diferentes tipos de projetos dentro da organização.

Preparar roteiro de entrevistas. A partir da seleção dos casos, o roteiro de entrevistas semiestruturado foi elaborado. Optou-se por dividir o roteiro de acordo com os grupos de processos do PMBoK (2013). Tomou-se como base o guia de entrevistas proposto por Eder (2012).

Realizar entrevistas. As entrevistas foram agendadas na empresa conforme disponibilidade dos respondentes. A duração média das entrevistas foi de 1 hora e o foco estava em identificar o conjunto de práticas (ações, técnicas e ferramentas) utilizadas na organização.

Analisar dados. A análise dos resultados ocorreu de forma qualitativa, uma vez que o interesse estava em interpretar as respostas dos respondentes e não na quantificação dessas. Essa atividade envolveu a transcrição e organização dos dados coletados em uma planilha eletrônica, conforme exemplo apresentado no Quadro 1. O objetivo foi analisar os dados a fim de identificar práticas em uso na empresa, além de padrões e diferenças entre essas. Os dados brutos foram ordenados e agrupados de acordo com a amostra entrevistada e processos de gerenciamento de projetos relacionados. Posteriormente, procurou-se eliminar práticas similares, a fim de evitar redundâncias.

Quadro 1. Estrutura utilizada na análise dos dados do estudo de caso. Fonte: Autoria própria.

\begin{tabular}{|c|c|c|c|c|c|c|}
\hline \multirow{2}{*}{ Cód } & \multirow{2}{*}{ Descrição } & \multicolumn{3}{|c|}{ Prática } & \multirow{2}{*}{$\begin{array}{c}\text { Processo } \\
\text { relacionado }\end{array}$} & \multirow{2}{*}{ Similaridade } \\
\hline & & Ação & Técnica & Ferramenta & & \\
\hline E3.6 & $\begin{array}{l}\text { Possuem um } \\
\text { cronograma com o } \\
\text { cliente que } \\
\text { envolve as } \\
\text { responsabilidades } \\
\text { macro da empresa } \\
\text { e do cliente, e um } \\
\text { cronograma } \\
\text { interno baseado } \\
\text { em sprints }\end{array}$ & $\begin{array}{l}\text { Desenvolver o } \\
\text { cronograma }\end{array}$ & $\begin{array}{l}\text { Product } \\
\text { Backlog/Sprint } \\
\text { Backlog/Gantt }\end{array}$ & $\begin{array}{l}\text { Software de } \\
\text { gerenciamento } \\
\text { de projetos }\end{array}$ & Planejamento & E. 4.5 e E. 2.4 \\
\hline
\end{tabular}

Além do estudo de caso, realizou-se uma revisão bibliográfica sobre modelos híbridos, a fim de fundamentar a presente pesquisa e sua discussão. Ao todo, doze propostas de modelos híbridos foram analisadas a fim de serem comparadas com as práticas identificadas na empresa estudada: a) XPrince (NAWROCKI et al., 2006); b) Mobile Software Development Methodology (RAHIMIAN; RAMSIN, 2008); c) Método híbrido de desenvolvimento de 
software (CHO, 2009); d) Modelo Híbrido Disciplinado (ZAKI; MOAWAD, 2010), e) Framework Tragile (SEYAM; GALAL-EDEEN, 2011); f) Disciplined Agile Delivery (AMBLER, 2013), g) Modelo Cocktail (BINDER; AILLAUD; SCHILLI, 2014), h) AgileStage-Gate (COOPER, 2014); i) Industrial Scrum Framework, (SOMMER et al., 2015); j) Planejamento de tempo combinado (SILVA, 2015); k) XSR Model (AHMAD; SOOMRO; BROHI, 2014); e l) IVPM2 (CONFORTO; AMARAL, 2016).

\section{RESULTADOS}

A análise dos dados permitiu compreender como o gerenciamento de projetos ocorre dentro da organização estudada. Os resultados obtidos estão apresentados no Quadro 2, fazendo um paralelo com o que os modelos da literatura defendem e o que foi identificado na empresa estudada. A fim de facilitar o entendimento, a apresentação das práticas está dividida em seis características específicas que distinguem as abordagens de gestão de projetos, adaptadas da proposta de Eder et al., (2015).

Observa-se que a empresa estudada possui diferentes tipos de práticas de gerenciamento em um mesmo ambiente. A empresa utiliza práticas e princípios tanto da teoria tradicional, quanto da teoria ágil, de forma conjunta, ficando evidente por exemplo com o uso de Status Report e do software MS-Project em conjunto com dispositivos visuais e cerimônias do Scrum.

Outro exemplo é o uso de um planejamento macro do projeto, de alto nível, onde se prepara um cronograma do projeto abordando os principais marcos e entregas do projeto, em conjunto com um planejamento de curto prazo (iteração), onde a equipe de projeto prioriza, escolhe e executa uma série de atividades a serem realizadas nesse período, a fim de gerar entregas parciais de valor para o cliente do projeto. Esse aliás, é um dos principais pontos defendidos pelos modelos híbridos da literatura. Há, portanto, evidências de uso de modelos híbridos, mesmo não sendo considerado pela empresa como tal.

Os modelos da literatura pregam em geral ações comuns como definir a missão e objetivos do projeto, listar valores centrais e propor uma arquitetura inicial, que são tratadas pela empresa estudada por meio das práticas relacionadas ao escopo do projeto como uso do Project Model Canvas, Service Model Canvas e documento de escopo do trabalho.

Outros aspectos semelhantes entre a empresa e os modelos da literatura está no controle do projeto. Os modelos ressaltam pontos específicos da abordagem orientada ao plano como documentação padronizada e revisões de fase, em conjunto com práticas ágeis, como uso de artefatos visuais, entregas parciais e atualização do plano do projeto. Essas ações foram identificadas na empresa analisada como comprovado pelo uso de relatórios em conjunto com dispositivos visuais, repositório de documentos em conjunto com cerimônias do Scrum, checkpoints mensais, entre outras.

Por outro lado, alguns aspectos presentes nos modelos da literatura são negligenciados na empresa analisada, como uso de técnicas de visão do produto e a elaboração de uma arquitetura física ainda em estágios iniciais de desenvolvimento a fim de refletir as principais funcionalidades do projeto. 
04 e 05 de setembro de 2017 - Escola Politécnica da Universidade de São Paulo

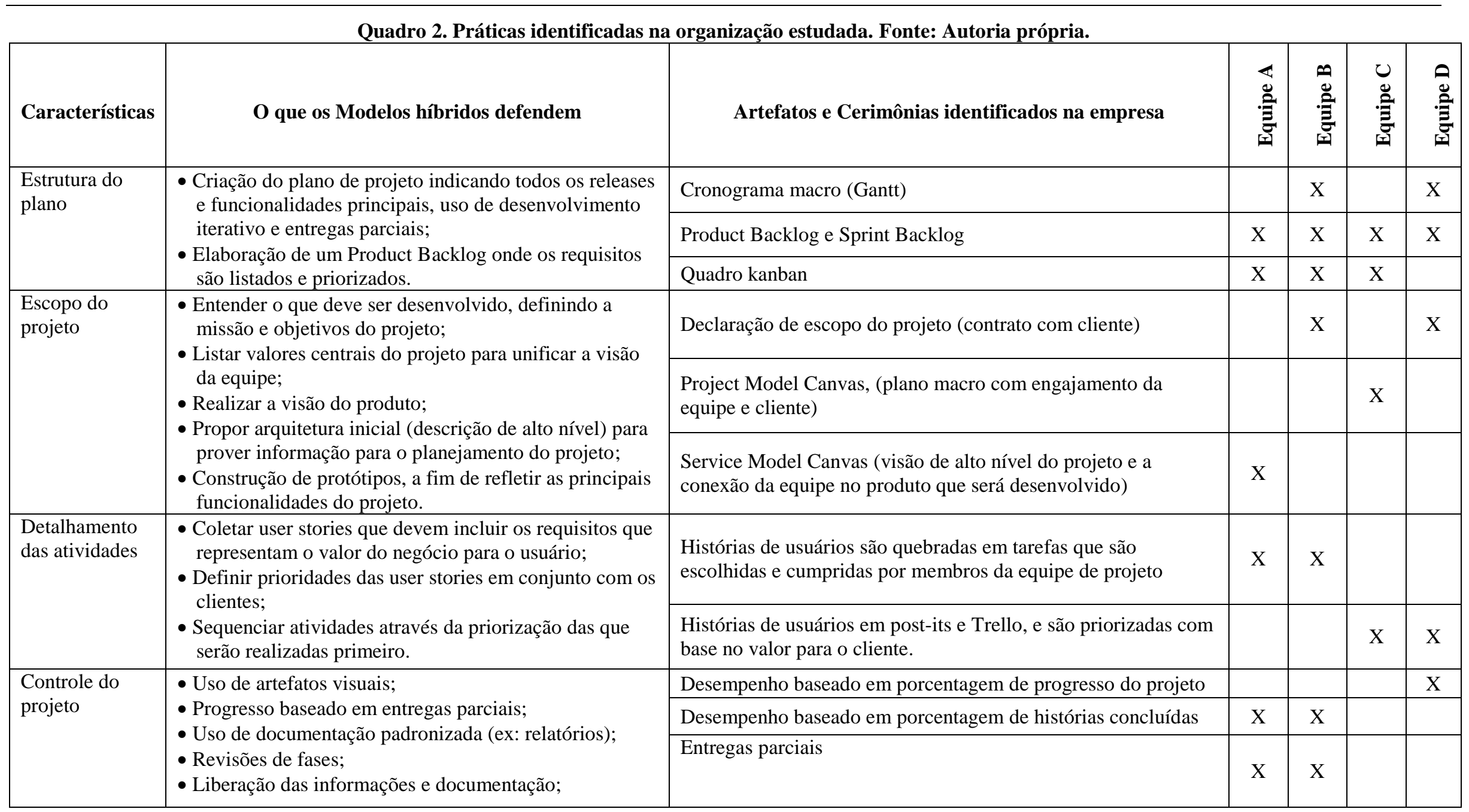




\begin{tabular}{|c|c|c|c|c|c|c|}
\hline Características & O que os Modelos híbridos defendem & Artefatos e Cerimônias identificados na empresa & 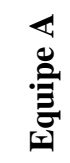 & 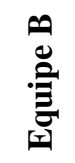 & 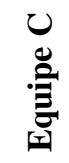 & : \\
\hline & \multirow{14}{*}{$\begin{array}{l}\text { - Atualização do plano de projeto a cada iteração; } \\
\text { - Predomínio de comunicação informal; } \\
\text { - Uso de sistema para gerenciamento de projetos; } \\
\text { - Uso de cerimônias do Scrum, como reuniões diárias e } \\
\text { reunião de retrospectiva. }\end{array}$} & Emprego de dispositivos visuais & $\mathrm{X}$ & $\mathrm{X}$ & & \\
\hline & & Net Promoter Score (NPS) para avaliar a satisfação do cliente & $\mathrm{X}$ & $\mathrm{X}$ & $\mathrm{X}$ & $\mathrm{X}$ \\
\hline & & Hapiness Canvas para avaliar satisfação da equipe & $\mathrm{X}$ & $\mathrm{X}$ & & \\
\hline & & Métricas do Kanban & $\mathrm{X}$ & & $\mathrm{X}$ & \\
\hline & & Lead time & $\mathrm{X}$ & & $\mathrm{X}$ & \\
\hline & & Throughput & $\mathrm{X}$ & & & \\
\hline & & Diagrama de fluxo cumulativo & $\mathrm{X}$ & & $\mathrm{X}$ & \\
\hline & & Relatórios de atividades & & & & $\mathrm{X}$ \\
\hline & & Status Report & & $\mathrm{X}$ & & \\
\hline & & Repositório de documentos com versionamento & $\mathrm{X}$ & $\mathrm{X}$ & $\mathrm{X}$ & $\mathrm{X}$ \\
\hline & & Software de gerenciamento de projetos & $\mathrm{X}$ & $\mathrm{X}$ & $\mathrm{X}$ & $\mathrm{X}$ \\
\hline & & Checkpoint mensal para discutir o andamento do projeto & $\mathrm{X}$ & & $\mathrm{X}$ & \\
\hline & & Reunião de status semanal com os gerentes de projetos & & & & $X$ \\
\hline & & Cerimônias do Scrum & $\mathrm{X}$ & $\mathrm{X}$ & $\mathrm{X}$ & \\
\hline \multirow{10}{*}{$\begin{array}{l}\text { Estratégia para } \\
\text { garantia do } \\
\text { atingimento do } \\
\text { escopo }\end{array}$} & \multirow{10}{*}{$\begin{array}{l}\text { - Definir os usuários do sistema e os respectivos } \\
\text { requisitos; } \\
\text { - Reuniões frequentes com cliente para coletar } \\
\text { feedbacks; } \\
\text { - Reunião de aceitação, onde a funcionalidade de todo o } \\
\text { sistema é avaliada pelo product owner e cliente. }\end{array}$} & $\begin{array}{l}\text { O cliente é envolvido no início do projeto para definição do } \\
\text { escopo }\end{array}$ & $\mathrm{X}$ & $\mathrm{X}$ & $\mathrm{X}$ & $\mathrm{X}$ \\
\hline & & O cliente tem uma interação periódica com a equipe de projeto & & $\mathrm{X}$ & $\mathrm{X}$ & \\
\hline & & O cliente está presente diariamente no projeto & $\mathrm{X}$ & & & \\
\hline & & O cliente tem uma interação técnica ao longo do projeto & & & & $\mathrm{X}$ \\
\hline & & Não há um encerramento presencial & $\mathrm{X}$ & & $\mathrm{X}$ & $\mathrm{X}$ \\
\hline & & A entrega final é realizada de forma presencial & & $X$ & & \\
\hline & & Não ocorre uma finalização formal & $\mathrm{X}$ & & $\mathrm{X}$ & \\
\hline & & Uso de e-mail & $\mathrm{X}$ & & $\mathrm{X}$ & $\mathrm{X}$ \\
\hline & & Uso de relatório final & & & & $\mathrm{X}$ \\
\hline & & O cliente sinaliza o fim do projeto com antecedência & $\mathrm{X}$ & & $\mathrm{X}$ & \\
\hline \multirow{3}{*}{$\begin{array}{l}\text { Estimativa de } \\
\text { recursos e } \\
\text { duração }\end{array}$} & \multirow{3}{*}{$\begin{array}{l}\text { - Atribuir estimativas de esforço necessárias para } \\
\text { concluir as histórias de usuários; } \\
\text { - Atribuir responsável para cada user story. }\end{array}$} & $\begin{array}{l}\text { A estimativa de duração é baseada em horas para cumprir uma } \\
\text { determinada atividade }\end{array}$ & $X$ & $\mathrm{X}$ & $\mathrm{X}$ & $X$ \\
\hline & & Estimativas paramétricas (estimativas de base histórica) & & $\mathrm{X}$ & & $\mathrm{X}$ \\
\hline & & Estimativa por opinião especializada, & $\mathrm{X}$ & & $\mathrm{X}$ & \\
\hline
\end{tabular}


Um ponto importante a ser ressaltado é que as propostas da literatura apresentam modelos gerais que devem ser aplicados a todos os projetos de uma organização, nos quais as abordagens puras não sejam suficientes para atender suas necessidades. Nenhuma das propostas analisadas apresenta um procedimento sistemático de como criar um modelo de gestão. Entretanto, conforme evidenciado no estudo, a empresa não segue um modelo predefinido, mas sim vários modelos que a própria organização criou a fim de atender as necessidades de seus projetos. Observa-se portanto, uma lacuna na teoria em relação a procedimentos capazes de auxiliar na combinação entre práticas orientadas ao plano e ágeis.

\section{CONSIDERAÇÕES FINAIS}

O estudo baseia-se na discussão sobre o tema gestão híbrida de projetos, apresentando um caso real na indústria de softwares, comparando as práticas e modelos híbridos utilizados pela empresa com os presentes na literatura, apontando semelhanças e diferenças. Tal tema vem crescendo nos últimos anos, e poucos são os estudos que discutem casos reais do uso de práticas hibridas em uma organização.

O estudo apresenta um registro de organização que combina métodos de gestão de projetos e identifica práticas alinhadas com os modelos híbridos de gestão presentes na literatura como o uso combinado de cronograma (Gantt) com painéis visuais e iterações. Identificou-se também aspectos que são defendidos pelos modelos da literatura, porém a empresa não emprega, como o uso de técnicas de visão do produto e de protótipos ainda em fases iniciais do projeto.

Outro resultado interessante é a constatação que os modelos de gestão utilizados pela empresa variam para diferentes tipos de projetos. Esse pode ser um indício que modelos predefinidos como os encontrados na literatura podem não satisfazer as necessidades dos projetos de uma organização, havendo a necessidade de adequar para cada projeto em específico, um modelo de gestão que satisfaça suas particularidades. A questão portanto, não seria escolher entre práticas de uma abordagem ou de outra, mas sim equilibrá-las conforme as características específicas do projeto e da organização. Entre essas características estão os níveis de complexidade, de inovação, estratégias, que se não forem levados em consideração, podem comprometer a qualidade e sucesso de um projeto.

Tais fatos comprovam a crescente busca das organizações por modelos de gestão capazes de atender os diferentes desafios, incertezas e complexidade do ambiente de negócios. Como pesquisas futuras, indica-se estudar procedimentos que auxiliem a escolha de práticas e personalização de modelos híbridos de gestão de projetos mais apropriadas a um determinado projeto, a fim de atender suas necessidades, estando alinhadas com suas características, aspectos organizacionais e características da equipe de projeto. 


\section{REFERÊNCIAS}

AHMAD, G.; SOOMRO, T.; BROHI, M. XSR: Novel Hybrid Software Development Model (Integrating XP, Scrum \& RUP). International Journal of Soft Computing and Engineering, $\mathrm{n}$. 3, p. 126-130, 2014.

AMARAL, D. C. et al. Gerenciamento Ágil de Projetos: Aplicação em Produtos Inovadores. Saraiva ed.São Paulo: Saraiva, 2011.

AMBLER, S. W. Going Beyond Scrum: Disciplined Agile Delivery. Disciplined Agile Consortium, White Paper Series, n. October, 2013.

BARLOW, J. B. et al. Overview and Guidance on Agile Development in Large Organizations. Communications of the Association for Information Systems, v. 29, n. July 2011, p. 25-44, 2011.

BATRA, D. et al. Balancing agile and structured development approaches to successfully manage large distributed software projects: A case study from the cruise line industry. Communications of the Association for Information Systems, v. 27, n. 1, p. 379-394, 2010.

BINDER, J.; AILLAUD, L. I.; SCHILLI, L. The Project Management Cocktail Model: An Approach for Balancing Agile and ISO 21500. Procedia - Social and Behavioral Sciences, v. 119, p. 182-191, 2014.

BOEHM, B. Get ready for agile methods, with care. Computer, v. 35, n. 1, p. 64-69, 2002.

BOEHM, B.; TURNER, R. Using risk to balance agile and plan-driven methods. Computer, v. 36, n. 6, p. 57-66, 2003a.

CHO, J. A hybrid software development method for large-scale projects: rational unified process with scrum. Issues in Information Systems, v. 10, n. 2, p. 340-348, 2009.

COBB, C. G. Making Sense of Agile Project Management: Balancing Control and Agility. New Jersey: John Wiley, 2012.

CONFORTO, E. C. et al. Can Agile Project Management Be Adopted by Industries Other than Software Development? Project Management Journal, v. 45, n. 3, p. 21-34, 2014.

CONFORTO, E. C.; AMARAL, D. C. Agile project management and stage-gate model-A hybrid framework for technology-based companies. Journal of Engineering and Technology Management, v. 40, p. 1-14, 2016.

CONFORTO, E.C.; SILVA, F.B.; AMARAL, D.; REBENTISCH, E. Modelos Híbridos unindo Complexidade, Agilidade e Inovação, Revista Mundo PM, v.70, n.2, 2015.

CONFORTO, E. C.; REBENTISCH, E.; AMARAL, D. C. The Building Blocks of Agility as a Team 's Competence. Project Management Agility Global Survey, 2014.

COOPER, R. G. What's Next?: After Stage-Gate. Research-Technology Management, v. 57, n. 1, p. 20-31, 2014.

COOPER, R. G. Agile-Stage-Gate Hybrids. Research Technology Management, v. 59, n. 1, p. 21-29, 2016.

COOPER, R. G.; SOMMER, A. F. The Agile-Stage-Gate Hybrid Model: A Promising New Approach and a New Research Opportunity. Journal of Product Innovation Management, v. 00, n. 00, p. n/a-n/a, 2016. 
COOPER, R. G. Idea-to-Launch Gating Systems : Better, Faster, and More Agile Idea-toLaunch Gating Systems. Research-Technology Management, v. 60, n. 1, p. 48-52, 2017.

EDER, S. Práticas de gerenciamento de projetos de escopo e tempo nas perspectivas das abordagens ágil e tradicional. 2012. 190 p. Dissertação (Mestrado) - Escola de Engenharia de São Carlos, Universidade de São Paulo, São Carlos, 2012.

EDER, S. et al. Diferenciando as abordagens tradicional e ágil de gerenciamento de projetos. Production Journal, v. 25, n. 3, p. 482-497, 2015.

GALAL-EDEEN, G.H.; RIAD, A.M.; SEYAM, M.S. Agility versus Discipline: is reconciliation possible? International Conference on Computer Engineering \& Systems (ICCES), Cairo, pg.331-337, November 2007.

KTATA, O.; LÉVESQUE, G. Agile development: Issues and avenues requiring a substantial enhancement of the business perspective in large projects. ACM, p. 59-66, 2009.

MARCONI, M.; LAKATOS, E. Fundamentos de metodologia científica. São Paulo, Editora Atlas, 2003.

MIGUEL, Paulo Augusto Cauchick et al. Estudo de caso na engenharia de produção: estruturação e recomendações para sua condução. Revista Produção, v. 17, n. 1, p. 216-229, 2007.

MIGUEL, Paulo A. C. et al. Metodologia de Pesquisa em Engenharia de Produção e Gestão de Operações. 2. ed. Rio de Janeiro: Elsevier, 2011.

NAWROCKI, J. et al. Balancing agility and discipline with XPrince. Lecture Notes in Computer Science, p. 266-277, 2006.

PROJECT MANAGEMENT INSTITUTE (PMI). A guide to the project management body of knowledge (PMBOK® guide) - 5th edition. Newtown Square, PA: Author, 2013.

RAHIMIAN, V.; RAMSIN, R. Designing an agile methodology for mobile software development: a hybrid method engineering approach. Research Challenges in Information Science, 2008.

SEYAM, M. S.; GALAL-EDEEN, G. H. Traditional versus Agile : The Tragile Framework for Information Systems Development. International Journal of Software Engineering (IJSE), v. 4, n. 1, p. 63-93, 2011.

SILVA, F. B.; AMARAL, D. C. Hibridismo na gestão de projetos: avaliando propostas de combinação das abordagens ágil e tradicional. In: XXXIV Encontro Nacional de Engenharia de Produção, 2014, Curitiba. Engenharia de Produção, Infraestrutura e Desenvolvimento Sustentável: a Agenda Brasil + 10, 2014. v. 1. p. 1-8.

SILVA, F. B. Proposta e avaliação de um procedimento de planejamento de tempo combinado ágil e tradicional. Dissertação (Mestrado) - Escola de Engenharia de São Carlos, Universidade de São Paulo, 2015.

SOMMER, A. F. et al. Improved Product Development Performance through Agile/Stage-Gate Hybrids. Research Technology Management, v. 58, n. 1, p. 34-44, 2015.

YIN, R. K. Estudo De Caso - Planejamento E Metodos. 2. ed. [s.1.] Bookman, 2001.

ZAKI, K. M.; MOAWAD, R. A Hybrid Disciplined Agile Software Process Model. The 7th International Conference on Informatics and Systems (INFOS), 2010. 